\title{
PRODUCTION AND REACTIVITY OF IONIZED CLUSTERS IN THE GAS PHASE
}

\author{
ANNA GIARDINI-GUIDONI and ALDO MELE \\ Universita "La Sapienza” P.Le A. Moro, 5 00185, Rome, Italy
}

\begin{abstract}
A brief review of the experimental methods to obtain gas phase cluster ions is reported. Supersonic expansion and ionization, high pressure ion production and clusterization, ablation from solids are techniques used to study cluster chemistry. Studies of cluster ion formation from metal, metal oxides and metal carbides are illustrated together with considerations on their structure and stability.
\end{abstract}

KEY WORDS: Laser, cluster, structure, ablation, ions, reactivity.

Clusters can be defined as an assembly of atoms and molecules sometimes attached to ions whose fractal structure is originated by the different types of forces linking the atoms and molecules. The term clusters is however used to describe collection of molecules on a surface, complexes existing in condensed phase, large inorganic and organometallic complexes as well as isolated assemblies in the gas phase. With such a broad definition it is not surprising that the literature devoted to the subject is enormous and pervades a wide range of fields involving both science and technology. ${ }^{1}$

Observation of gas-phase clusters have been obtained by several techniques. The experimental systems for effecting the desired degree of aggregation will be briefly mentioned here.

\section{Ionization of Neutral Clusters Produced by Supersonic Expansion}

The most widely used method is the adiabatic expansion of a gas or a vapor through a small nozzle into vacuum. ${ }^{2}$ Expansion is often accomplished in combination with an inert carrier gas which serves to assist in the cooling and stablization of clusters. Ionization is obtained by passing the cluster beam through some ionizing source.

\section{High Pressure Gas Phase Ionization}

Another method which results in the formation of cluster ions involves the creation of ions in gaseous media. In this case primary ions evolve into clusters through a series of chemical reactions including clustering with the surrounding gas. $^{3}$ 


\section{Vaporization of Solids}

Clusters from solids can be easily obtained by sputtering techniques. These techniques are based on depositing energy through ions, electrons or photons onto a surface which results in ablation and ionization of a solid target. The mechanism of cluster ion formation in sputtering processes is not yet clear. The numerous studies have generally not been able to clearly differentiate among various possible processes. ${ }^{4}$ The techniques which involve the bombardment of surfaces by atoms and ions are commonly called Fast Atom Bombardment (FAB) and Secondary Ions Mass Spectrometry (SIMS) respectively. Another method, utilized in our laboraory, is vaporization by lasers emitting in the IR or VIS UV region and is called laser ablation mass spectrometry (LAMS). ${ }^{5}$

As for gas phase cluster ions the questions to answer concern: chemical and structural identity, and chemical reactivity. Mass spectrometry and mass spectral abundance pattern can be interpreted as being indicative of cluster identity and structure. In many cases the so called "magic numbers" observed have been compared with "theoretical" predictions obtained by using electronic or structural models. Production of large size mixed clusters gives information on chemical reactivity. Finally the analysis of the deposits they originate may be of help in some cases. The matter is quite large and this review will report only a few studies on simple metal clusters and their oxides and carbides to show what type of information is usually obtained.

\section{Metal Clusters}

The observation of prominent features in the abundance spectra of metal clusters, in particular of sodium, ${ }^{6}$ prompted the examination of the data with a simple shell model. The stability of alkali metal clusters has been described in terms of an electronic shell closing model which describes the cluster as a structureless ionic jelly. ${ }^{7}$ The hypothesis is that in the metal the electrons move rather freely in an almost spherically symmetrical environment, so that the shell structure appears to be of electronic origin. The main objective is to deal with the properties of electrons in these clusters whatever the cluster size may be. In the jellium model, the effect of ionic cores is simulated by a uniformly positive charged background; wave functions and eigenvalues can be computed on a simple basis and the total energy $E(n)$ of the $n$-atom cluster can be calculated for each $n$.

The calculated results within the model for clusters of monovalent alkali metal indicate that the total energy per atom $E(n) / n$ shows small features at $n=8,18,20$, $34,40,58,92$ where the total energy appears to change abruptly. The theoretical calculations on $\mathrm{Na}$ clusters matches quite well with the experimental data. This model has been applied successfully to other metals. Experimental abundance spectra correlates with this shell model for $\mathrm{Mg}, \mathrm{Al}, \mathrm{Au}, \mathrm{Cu}$ and $\mathrm{Ag}$.

\section{Metal Oxides}

Quite interesting "per se" and for the implications as precursors of high temperature superconductors are the studies concerning metal oxides. Numerous simple oxides 
have been studied in our and other laboratories. ${ }^{5}$ In our laboratory the laser ablation of solid samples and time of flight mass analysis technique has been applied. The mass spectra are characterized by positively and negatively charged oxide cluster ions of different composition up to a high number of atoms. The trend of the intensity of the mass spectra show preferred abundance of series of homologous compounds. The composition of the clusters is in some cases different to the stoichiometry of the target compounds. Typically the data of calcium oxide show one series corresponding to the stoichiometry solid oxide target $(\mathrm{CaO}) n$ together with another sequence of metal rich oxide sequences $\mathrm{Ca}(\mathrm{CaO}) n$. This behaviour is characteristic of other II A group oxides: $\mathrm{Mg}, \mathrm{Sr}$ and Ba oxide. A similar trend has been observed in SIMS and in seeded molecular beam expansion experiments. ${ }^{8}$

Structure and stability of these clusters can be qualitatively explained by using an ionic model similar to that employed to explain many of the features observed in the mass spectra of alkali halide clusters. ${ }^{9,10}$ By this simple approach the intensity anomalies or magic cluster numbers are easy to understand when an ionic interaction potential is assumed and the stable clusters can be thought to be composed of $\mathrm{Ca}^{2+}$ and $\mathrm{O}^{2-}$ closed shell ions. The abundance maxima observed in the series are explained as arising from the exceptional stability of compact cubic structures that are essentially pieces of the face centered cubic (f.c.c.) metal oxide crystal lattice.

The series corresponding to metal rich oxide $\mathrm{Me}(\mathrm{MeO})^{+}{ }_{n}$, which is characteristic of all oxide metal clusters, has been explained on the basis of the ionic model as leading to abundance maxima when $n$ is one less than the maxima of stoichiometric clusters. In fact in the cubic structure the excess electron from the extra metal atoms could be localized in an anion vacancy in the lattice analogous to a solid state $F$ center. ${ }^{9}$

A feature shown by a large part of the positive cluster ions formed by all other oxides seems of particular interest for the interpretation of the cluster stability and structure. It has been found that besides cluster ions of the stoichiometric composition several series are characterized by the presence of $\mathrm{Me}$ or $\mathrm{MeO}$. Examples are Scandium, Lanthanum and Yttrium oxide ion intensities. ${ }^{5}$ A qualitative description of the cluster formed and of their stability may be attempted. The non-stoichiometric composition of some oxide cluster ion series would suggest that the structures of oxide cluster ions may be defective and this may be more pronounced for clusters of low mass, while the high molecular weight cluster composition is essentially characterised by the valence of the element forming the oxides; the ratio between bulk and surface atoms may play a relevant role on the stability of cluster. It seems evident that high molecular weight clusters reproduce the structure of the solid and bulk atoms prevail with respect to surface structure. The smaller the cluster, the more important is the role of surface energy, since surface atoms represent a large portion of the total.

\section{Carbides}

Laser evaporation of a mixture of III B group oxides and graphite yields ionized metal carbide cluster of formula $\mathrm{Me}_{\mathrm{m}} \mathrm{C}_{\mathrm{n}}{ }^{+} .{ }^{12}$ The interesting feature of the mass 
spectrum of carbide clusters is the even-odd alternation already found in carbon clusters. This result can be interpreted analogously to bare carbon cluster stability, at least when the number of carbon atoms is lower than seven. Up to this number a variable stoichiometry between metal and carbon atoms is also seen and this suggests that in this range carbide clusters may be analogous in structure of carbon aggregates with metal atoms alternating or at the terminal of the carbon chain. For $n$ larger than seven the ratio $m: n$ of metal to carbon tends to two indicating that larger clusters are characterized by a metal content defined by a precise ratio.

\section{Mixed Oxides}

This brief review concludes by showing the links between cluster field and the field of semi and super conductive materials. The reactions of mixture and super conductive materials. The reactions of mixtures of solid oxides have been investigated by laser irradiation. ${ }^{5}$ Oxide cluster ions of two or more components in various ratios have been produced. In particular the study has been focused on oxide precursors of copper based superconductor oxides. Complex cluster ions, formed when copper oxide is mixed with other oxides, are present in the mass spectra as well as in the laser ablated superconductor. This study has shown that chemical reactions take place in the process of laser evaporation of the solid target. These data indicate that the $\mathrm{Cu}^{+}$ ion always reacts with oxide clusters to give a $\mathrm{Cu}$ rich composite oxide ion. This fact can be linked to the role of copper in superconducting material.

\section{References}

1. A. W. Castleman and R. G. Keesee, Chem. Rev. 86, 589 (1986); (b) J. C. Phyllips, Chemn. Rev. 86, 618 (1986); (c) A. Kaldor, D. M. Cox and M. R. Zakin, Advances Chem. Phys. LXX, 2, p. 211 (1988).

2. O. F. Hagena, Surf. Sci. 106, 101 (1981).

3. (a) P. Kebarle, Annu. Rev. Phys. Chem. 28, 445 (1977); (b) A. Giardini-Guidoni, A. Mele, F. Vecchiocattivi and F. Zocchi, Trans. Faraday Soc. 66, 1464 (1970).

4. A. Vertes, P. Juhasz, M. DeWolf and R. Gijbels, Scanning Microscopy Int. 2(4), 1853 (1968).

5. (a) A. Mele, D. Consalvo, D. Stranges, A. Giardini-Guidoni and R. Teghil, Int. J. Mass. Spect. and Ion Processes 95, 359 (1990); (b) E. Michels and R. Gijbels, Anal. Chem. 56, 1115 (1984).

6. M. Y. Chou, A. Cleland and M. L. Cohen, Solid State Commun. 52, 645 (1984); (b) C. Brechignac, Ph. Cahuzac, J. Leygnier, R. Pflaum, J. Ph. Roux and J. Weiner, Z. Phys. D. Atoms, Moleculea and Clusters 12, 199 (1989).

7. W. A. Knight, K. Clemenger, W. A. de Heer, W. A. Sauniders, M. Y. Chou and M. L. Cohen, Phys. Rev. Letters 52, 2141 (1984).

8. W. A. Deheer, W. D. Knight, M. Y. Chou and M. L. Cohen, Solid State Physics 40, 93 (1987) and references therein.

9. P. J. Zieman and A. W. Castleman, J. Chem. Phys. (in press).

10. T. P. Martin, Phys. Rep. 95, 167 (1983).

11. R. Pflaum, P. Pfan, K. Sattler and E. Rechnagel, Surf Sci. 156, 165 (1985).

12. A. Giardini-Guidoni, A. Mele, G. Pizzella and R. Teghil, Z. Phys. D. Atoms, Molecules and Clusters 20, 89-91 (1991). 